\title{
A COUNTEREXAMPLE TO A MULTILINEAR ENDPOINT QUESTION OF CHRIST AND KISELEV
}

\author{
Camil Muscalu, Terence Tao, and Christoph Thiele
}

\begin{abstract}
Christ and Kiselev [2],[3] have established that the generalized eigenfunctions of one-dimensional Dirac operators with $L^{p}$ potential $F$ are bounded for almost all energies for $p<2$. Roughly speaking, the proof involved writing these eigenfunctions as a multilinear series $\sum_{n} T_{n}(F, \ldots, F)$ and carefully bounding each term $T_{n}(F, \ldots, F)$. It is conjectured that the results in [3] also hold for $L^{2}$ potentials $F$. However in this note we show that the bilinear term $T_{2}(F, F)$ and the trilinear term $T_{3}(F, F, F)$ are badly behaved on $L^{2}$, which seems to indicate that multilinear expansions are not the right tool for tackling this endpoint case.
\end{abstract}

\section{Introduction}

Let $F(x)$ be a real potential on $\mathbb{R}$. For each energy $k^{2}>0$ we can consider the Dirac generalized eigenfunction equation

$$
\left(\frac{d}{d x}+F\right)\left(-\frac{d}{d x}+F\right) \phi(x)=k^{2} \phi(x)
$$

on $\mathbb{R}$. This Dirac equation can be thought of as a Schrödinger equation with potential $V=F^{\prime}+F^{2}$. For each $k$ there are two linearly independent eigenfunctions $\phi=\phi_{k}$. A natural question from spectral theory is to ask whether these eigenfunctions are bounded (i.e. are in $L_{x}^{\infty}$ ) for almost every real $k$. In [3] Christ and Kiselev ${ }^{1}$ showed among other things that this was true when $F \in L_{x}^{p}$ for any $1 \leq p<2$. It is well known (see e.g. [12]) that the statement fails when $p>2$, but the $p=2$ case remains open. In [5] it is shown that for $L^{2}$ potentials one has absolutely continuous spectrum on $[0, \infty)$, but this is a slightly weaker statement.

We briefly outline the arguments in [2],[3]. The method of variation of constants suggests the ansatz

$$
\begin{aligned}
\phi(x) & =a(x) e^{i k x}+b(x) e^{-i k x} \\
\left(-\frac{d}{d x}+F\right) \phi(x) & =-i k a(x) e^{i k x}+i k b(x) e^{-i k x} .
\end{aligned}
$$

Received July 30, 2001.

${ }^{1}$ The results cited are phrased for Schrödinger operators but also extend to the slightly simpler case of Dirac operators, see [4]. 
Substituting this into the previous and simplifying, we reduce to the first-order system

$$
\begin{aligned}
& a^{\prime}(x)=F(x) e^{-2 i k x} b(x) \\
& b^{\prime}(x)=F(x) e^{2 i k x} a(x) .
\end{aligned}
$$

For simplicity we may assume $F$ is supported on the positive half axis. If we set initial conditions $a(-\infty)=1, b(-\infty)=0$ for instance, and then solve this system by iteration, we thus obtain the formal multilinear expansions

$$
a=1+\sum_{n \geq 2, \text { even }} T_{n}(F, \ldots, F) ; \quad b=\sum_{n \geq 1, \text { odd }} T_{n}(F, \ldots, F)
$$

where for each $n \geq 1, T_{n}$ is the $n$-linear operator

$$
\begin{aligned}
& T_{n}\left(F_{1}, \ldots, F_{n}\right)(k, x):= \\
& \qquad \int_{x_{1}<\ldots<x_{n}<x} e^{-2 i k \sum_{j=1}^{n}(-1)^{j} x_{j}} F_{1}\left(x_{1}\right) \ldots F_{n}\left(x_{n}\right) d x_{1} \ldots d x_{n} .
\end{aligned}
$$

For integrable $F_{j}$ we can define the $n$-linear operators

$$
\begin{aligned}
& T_{n}\left(F_{1}, \ldots, F_{n}\right)(k,+\infty):= \\
& \qquad \int_{x_{1}<\ldots<x_{n}} e^{-2 i k \sum_{j=1}^{n}(-1)^{j} x_{j}} F_{1}\left(x_{1}\right) \ldots F_{n}\left(x_{n}\right) d x_{1} \ldots d x_{n} .
\end{aligned}
$$

The strategy of Christ and Kiselev was then to control each individual expression $T_{n}$ on $L^{p}$. Specifally, they showed the estimate

$$
\left\|\sup _{x}\left|T_{n}(F, \ldots, F)(k, x)\right|\right\|_{L_{k}^{p^{\prime} / n, \infty}} \leq C_{p, n}\|F\|_{L_{x}^{p}}^{n}
$$

for all $n \geq 1$ and $1 \leq p<2$, where $C_{p, n}$ was a constant which decayed rapidly in $n$ and $1 / p+1 / p^{\prime}:=1$. In particular one has the non-maximal variant

$$
\left\|T_{n}(F, \ldots, F)(k,+\infty)\right\|_{L_{k}^{p^{\prime} / n, \infty}} \leq C_{p, n}\|F\|_{L_{x}^{p}}^{n} .
$$

The boundedness of eigenfunctions for almost every $k$ then follows by summing these bounds carefully.

It is tempting to try this approach for the endpoint $p=2$. For $n=1$ we see that $T_{1}(F)(k,+\infty)$ is essentially the Fourier co-efficient $\hat{F}(k)$, while $\sup _{x}\left|T_{1}(F)(k, x)\right|$ is essentially the Carleson maximal operator $C F(k)$. The estimates (2), (1) for $p=2$ then follow from Plancherel's theorem and the Carleson-Hunt theorem [1], [6] respectively.

For $n=2$ the expression $T_{2}(F, F)(k,+\infty)$ is essentially $H_{-}\left(|\hat{F}|^{2}\right)(k)$, where $H_{-}$is the Riesz projection

$$
\widehat{H_{-} F}:=\chi_{(-\infty, 0]} \widehat{F},
$$

and so (2) follows for $p=2$ by Hölder's inequality and the weak-type $(1,1)$ of the Riesz projections. We also remark that if the phase function $x_{1}-x_{2}$ in the definition of $T_{2}$ were replaced by $\alpha_{1} x_{1}+\alpha_{2} x_{2}$ for generic numbers $\alpha_{1}, \alpha_{2}$ 
then the operator is essentially a bilinear Hilbert transform and one still has boundedness from the results in [7], [8], [13].

It may thus appear encouraging to try to estimate the higher order multilinear operators for $L^{2}$ potentials $F$. However, in this note we show

Theorem 1.1. When $p=2$ and $n=2$, the estimate (1) fails. When $p=2$ and $n=3$, the estimate (2) fails.

Because of this, we believe that it is not possible to prove the almost everywhere boundedness of eigenfunctions for Dirac or Schrödinger operators with $L^{2}$ potential purely by multilinear expansions; we discuss this further in the remarks section.

The counterexample has a logarithmic divergence, and essentially relies on the fact that while convolution with the Hilbert kernel $p \cdot v \cdot \frac{1}{x}$ is bounded, convolution with $\frac{\operatorname{sgn}(x)}{x}$ or $\frac{\chi_{(-\infty, 0]}(x)}{x}$ is not. It may be viewed as an assertion that $L^{2}$ potentials create significant long-range interaction effects which are not present for more rapidly decaying potentials.

Interestingly, our counterexamples rely strongly on a certain degeneracy in the phase function $\sum_{j}(-1)^{j} x_{j}$ on the boundary of the simplex $x_{1}<\ldots<x_{n}$. If one replaced this phase by $\sum_{j} x_{j}$, then we have shown in [9], [10] that the bound (2) in fact holds when $p=2$ and $n=3$. Indeed this statement is true for generic phases of the form $\sum_{j} \alpha_{j} x_{j}$. A similar statement holds for (1) when $p=2$ and $n=2$ and will appear elsewhere.

\section{Proof of Theorem 1.1}

The letter $C$ may denote different large constants in the sequel. To be consistent with the previous notation we shall define the Fourier transform as

$$
\hat{F}(k):=\int e^{-2 i k x} F(x) d x .
$$

We let $N \gg 1$ be a large integer parameter, which we shall take to be a square number, and test (1), (2) with the real-valued potential

$$
F(x):=\sum_{j=N}^{2 N} F_{j}(x)
$$

where the $F_{j}$ are given by

$$
F_{j}(x):=N^{-1} \cos \left(2 \frac{A j}{N} x\right) \phi\left(\frac{x}{N}-j\right),
$$

$\phi$ is a smooth real valued function supported in $\left[-\frac{1}{4}, \frac{1}{4}\right]$ with total mass $\int \phi=1$ such that $\widehat{\phi}$ stays away from 0 in $[-1,1]$, and $A$ is a sufficiently large absolute constant whose purpose is to ensure that

$$
4 \sum_{j \in \mathbb{Z} \backslash\{0\}}|\widehat{\phi}(\xi-A j)| \leq|\widehat{\phi(\xi)}|
$$


for $\xi \in[-1,1]$. Informally, $F$ is a "chirp" which is localized in phase space to the region

$$
\left\{(k, x): k= \pm \frac{A j}{N}+O\left(\frac{1}{N}\right) ; x=N j+O(N), N \leq j \leq 2 N\right\} .
$$

We may compute the Fourier transform of the $F_{j}$ using the rapid decay of $\hat{\phi}$ as

$$
\hat{F}_{j}(k)=\frac{1}{2} e^{-2 i(N k-A j) j} \hat{\phi}(N k-A j)+O\left(N^{-200}\right)
$$

in the region $\frac{A}{2}<k<3 A$. We remark that the error term $O\left(N^{-200}\right)$ has a gradient which is also $O\left(N^{-200}\right)$.

Clearly we have $\left\|F_{j}\right\|_{2}=O\left(N^{-1 / 2}\right)$, and hence that

$$
\|F\|_{2}=O(1) \text {. }
$$

We now compute

$$
T_{2}(F, F)(k, x)=\int_{x_{1}<x_{2}<x} e^{2 i k\left(x_{1}-x_{2}\right)} F\left(x_{1}\right) F\left(x_{2}\right) d x_{1} d x_{2}
$$

in the region

$$
\left|N k-A j_{0}\right| \leq 1 ; \quad x=N\left(j_{0}-\sqrt{N}+\frac{1}{2}\right)
$$

for some integer $\frac{3 N}{2}<j_{0}<2 N$. In this region we show that

$$
\left|T_{2}(F, F)(k, x)\right| \geq C^{-1} \log N,
$$

which will imply that

$$
\left\|\sup _{x}\left|T_{2}(F, F)\right|(k, x)\right\|_{L_{k}^{2, \infty}} \geq C^{-1} \log N
$$

and thus contradict (1) for $n=2$ and $p=2$ by letting $N$ go to infinity.

We now prove (6). Fix $k, j_{0}, x$. Observe from (4) that $T_{2}\left(F_{j}, F_{j^{\prime}}\right)(k, x)$ vanishes unless $j \leq j^{\prime} \leq j_{0}-\sqrt{N}$. Thus we may expand

$$
\begin{aligned}
T_{2}(F, F)(k, x) & =\sum_{N \leq j \leq j_{0}-\sqrt{N}} T_{2}\left(F_{j}, F_{j}\right)(k, x) \\
& +\sum_{N \leq j<j^{\prime} \leq j_{0}-\sqrt{N}} T\left(F_{j}, F_{j^{\prime}}\right)(k, x) .
\end{aligned}
$$

We first dispose of the error term (8). In the region $j<j^{\prime} \leq j_{0}-\sqrt{N}$, the conditions $x_{1}<x_{2}<x$ in (4) become superfluous, so we may factor

$$
T_{2}\left(F_{j}, F_{j^{\prime}}\right)(k, x)=\overline{\hat{F}_{j}(k)} \hat{F}_{j^{\prime}}(k) .
$$

However, since $\hat{\phi}$ is rapidly decreasing and $\left|j-j_{0}\right|,\left|j^{\prime}-j_{0}\right| \geq \sqrt{N}$, we see from (3) that

$$
\left|\hat{F}_{j}(k)\right|,\left|\hat{F}_{j^{\prime}}(k)\right| \leq C N^{-100} .
$$

Summing this, we see that the total contribution of $(8)$ is $O\left(N^{-198}\right)$. 
Now we consider the contribution of (7). We use the identity

$$
T_{2}\left(F_{j}, F_{j}\right)(k, x)=T_{2}\left(F_{j}, F_{j}\right)(k,+\infty)=H_{-}\left(\left|\hat{F}_{j}\right|^{2}\right)(k)
$$

combined with (3). The operator $H_{-}$is a non-trivial linear combination of the identity and the Hilbert transform, while $\left|\hat{F}_{j}\right|^{2}$ is essentially a non-negative bump function rapidly decreasing away from the interval $[j A / N-O(1 / N), j A / N+$ $O(1 / N)]$. Because of this we see that for $j \neq j_{0}$ we have

$$
H_{-}\left(\left|\hat{F}_{j}\right|^{2}\right)(k)=\frac{c}{j-j_{0}}+O\left(\left|j-j_{0}\right|^{-2}\right)
$$

where $c$ is a non-zero absolute constant. Summing this over all $j \leq j_{0}-\sqrt{N}$ and observing that $j-j_{0}$ has a consistent sign we see that the contribution of (7) has magnitude at least $C^{-1} \log N$, and (6) follows.

We now compute $T_{3}(F, F, F)(k,+\infty)$ in the region

$$
\left|N k-A j_{0}\right| \leq 1 ; \quad 1.4 N<j_{0}<1.6 N .
$$

We will show that

$$
\left|T_{3}(F, F, F)(k,+\infty)\right| \geq C^{-1} \log N
$$

in this region, which will disprove (2) for $n=3$ and $p=2$ similarly to before.

It remains to prove $(12)$. Fix $j_{0}$. Observe that $T_{3}\left(F_{j}, F_{j^{\prime}}, F_{j^{\prime \prime}}\right)(k,+\infty)$ vanishes unless $j \leq j^{\prime} \leq j^{\prime \prime}$. Thus we can split

$$
\begin{aligned}
T_{3}(F, F, F)(k,+\infty) & =\sum_{N \leq j \leq 2 N} T_{3}\left(F_{j}, F_{j}, F_{j}\right)(k,+\infty) \\
& +\sum_{N \leq j<j^{\prime} \leq 2 N} T_{3}\left(F_{j}, F_{j}, F_{j^{\prime}}\right)(k,+\infty) \\
& +\sum_{N \leq j^{\prime}<j \leq 2 N} T_{3}\left(F_{j^{\prime}}, F_{j}, F_{j}\right)(k,+\infty) \\
& +\sum_{N \leq j<j^{\prime}<j^{\prime \prime} \leq 2 N} T_{3}\left(F_{j}, F_{j^{\prime}}, F_{j^{\prime \prime}}\right)(k,+\infty) .
\end{aligned}
$$

We first consider (13). We expand

$$
T_{3}\left(F_{j}, F_{j}, F_{j}\right)(k,+\infty)=\int_{x_{1}<x_{2}<x_{3}} e^{2 i k\left(x_{1}-x_{2}+x_{3}\right)} F_{j}\left(x_{1}\right) F_{j}\left(x_{2}\right) F_{j}\left(x_{3}\right) d x_{1} d x_{2} d x_{3} .
$$

This is a linear combination of eight terms of the form

$$
\begin{aligned}
& N^{-3} \int_{x_{1}<x_{2}<x_{3}} e^{2 i k\left(x_{1}-x_{2}+x_{3}\right)} e^{2 i \frac{A j}{N}\left( \pm x_{1} \pm x_{2} \pm x_{3}\right)} \\
& \cdot \phi\left(\frac{x_{1}}{N}-j\right) \phi\left(\frac{x_{2}}{N}-j\right) \phi\left(\frac{x_{3}}{N}-j\right) d x_{1} d x_{2} d x_{3}
\end{aligned}
$$

making the substitutions $y_{s}:=\frac{x_{s}}{N}-j$ for $s=1,2,3$, this becomes

$$
e^{i \theta} \int_{y_{1}<y_{2}<y_{3}} e^{2 i k N\left(y_{1}-y_{2}+y_{3}\right)} e^{2 i A j\left( \pm y_{1} \pm y_{2} \pm y_{3}\right)} \phi\left(y_{1}\right) \phi\left(y_{2}\right) \phi\left(y_{3}\right) d y_{1} d y_{2} d y_{3}
$$


for some phase $e^{i \theta}$ depending on all the above variables.

We shall only consider the choice of signs $\left(-y_{1}+y_{2}-y_{3}\right)$; the reader may easily verify that the other choices of signs are much smaller thanks to stationary phase. In this case we can write the above as

$$
e^{i \theta} \int_{y_{1}<y_{2}<y_{3}} e^{2 i(k N-A j)\left(y_{1}-y_{2}+y_{3}\right)} \phi\left(y_{1}\right) \phi\left(y_{2}\right) \phi\left(y_{3}\right) d y_{1} d y_{2} d y_{3} .
$$

If $k N-A j=O(1)$ we estimate this crudely by $O(1)$. Otherwise we can perform the $y_{1}$ integral using stationary phase to obtain

$$
e^{i \theta} \frac{1}{2 i(k N-A j)} \int_{y_{2}<y_{3}} e^{2 i(k N-A j) y_{3}} \phi\left(y_{2}\right) \phi\left(y_{2}\right) \phi\left(y_{3}\right) d y_{2} d y_{3}+O\left(|k N-A j|^{-2}\right) \text {. }
$$

Performing another stationary phase we see that this quantity is $O\left(|k N-A j|^{-2}\right)$. Summing over all $j$ we see that $(13)$ is $O(1)$.

Let us now consider (16). When $j<j^{\prime}<j^{\prime \prime}$, the constraints $x_{1}<x_{2}<x_{3}$ in the definition of $T_{3}$ are redundant, and we can factorize

$$
T_{3}\left(F_{j}, F_{j^{\prime}}, F_{j^{\prime \prime}}\right)(k,+\infty)=\overline{\hat{F}_{j}(k)} \hat{F}_{j^{\prime}}(k) \overline{\hat{F}_{j^{\prime \prime}}(k)} .
$$

Applying (3) and using the rapid decay of $\hat{\phi}$ we see that

$$
\left|T_{3}\left(F_{j}, F_{j^{\prime}}, F_{j^{\prime \prime}}\right)(k,+\infty)\right| \leq C\left(1+\left|j-j_{0}\right|+\left|j^{\prime}-j_{0}\right|+\left|j^{\prime \prime}-j_{0}\right|\right)^{-10}+C N^{-100} .
$$

Summing over all $j, j^{\prime}, j^{\prime \prime}$ we see that (16) is $O(1)$.

It remains to control $(15)+(14)$. First we consider (14). For this term the condition $x_{2}<x_{3}$ is redundant, so we can factorize

$$
T_{3}\left(F_{j}, F_{j}, F_{j^{\prime}}\right)(k,+\infty)=T_{2}\left(F_{j}, F_{j}\right)(k,+\infty) \overline{\hat{F}_{j^{\prime}}(k)} .
$$

Now consider (15). For this term the condition $x_{1}<x_{2}$ is redundant, so we can factorize

$$
T_{3}\left(F_{j^{\prime}}, F_{j}, F_{j}\right)(k,+\infty)=\overline{\hat{F}_{j^{\prime}}(k)} \int_{x_{2}<x_{3}} e^{2 i k\left(x_{3}-x_{2}\right)} F_{j}\left(x_{2}\right) F_{j}\left(x_{3}\right) d x_{3} d x_{2} .
$$

Writing $x_{1}$ instead of $x_{3}$ we thus have

$$
T_{3}\left(F_{j^{\prime}}, F_{j}, F_{j}\right)(k,+\infty)=\overline{\hat{F}_{j^{\prime}}(k)}\left(\left|\hat{F}_{j}(k)\right|^{2}-T_{2}\left(F_{j}, F_{j}\right)(k,+\infty)\right) .
$$

Combining this with the previous, we thus see that

$$
\sum_{N \leq j, j^{\prime} \leq 2 N} \operatorname{sgn}\left(j^{\prime}-j\right) T_{2}\left(F_{j}, F_{j}\right)(k,+\infty) \overline{\hat{F}_{j^{\prime}}(k)}+\sum_{N \leq j^{\prime}<j \leq 2 N} \overline{\hat{F}_{j^{\prime}}(k)}\left|\hat{F}_{j}(k)\right|^{2} .
$$

Using (3) as in (16) we see the second term is $O(1)$, so to prove (12) it will suffice to show that

$$
\left|\sum_{N \leq j, j^{\prime} \leq 2 N, j \neq j^{\prime}} \operatorname{sgn}\left(j^{\prime}-j\right) T_{2}\left(F_{j}, F_{j}\right)(k,+\infty) \overline{\hat{F}_{j^{\prime}}(k)}\right| \geq C^{-1} \log N .
$$


We first consider the terms with $j^{\prime}=j_{0}$. We claim these terms are the dominant contribution. From (9), (10) we conclude

$$
\begin{aligned}
& \sum_{N \leq j \leq 2 N, j \neq j_{0}} \operatorname{sgn}\left(j_{0}-j\right) T_{2}\left(F_{j}, F_{j}\right)(k,+\infty) \overline{\hat{F}_{j_{0}}(k)}= \\
& \quad \sum_{N \leq j \leq 2 N, j \neq j_{0}} c \frac{\operatorname{sgn}\left(j_{0}-j\right)}{j_{0}-j} \overline{\hat{F}_{j_{0}}(k)}+O(1) .
\end{aligned}
$$

Here $c$ is the same non-zero constant as in $(10)$, and $\hat{F}_{j_{0}}(k)$ is bounded away from 0 by choice of $\phi$. Thus the first term is greater than $C^{-1} \log N$, so it suffices indeed to show that this term is the dominant contribution to (17).

We consider the terms with $j=j_{0}$. Using that $\left|T_{2}\left(F_{j}, F_{j}\right)(k, \infty)\right| \leq C$ we obtain

$$
\sum_{N \leq j^{\prime} \leq 2 N, j_{0} \neq j^{\prime}}\left|T_{2}\left(F_{j_{0}}, F_{j_{0}}\right)(k,+\infty) \overline{\hat{F}_{j^{\prime}}(k)}\right| \leq C
$$

This term is therefore negligible.

Finally, we have to consider the terms with $j, j^{\prime} \neq j_{0}$. We have by the choice of $A$,

$$
\begin{aligned}
& \sum_{N \leq j, j^{\prime} \leq 2 N, j, j^{\prime} \neq j_{0}}\left|T_{2}\left(F_{j}, F_{j}\right)(k,+\infty)\right|\left|\overline{\hat{F}_{j^{\prime}}(k)}\right| \\
\leq & \frac{1}{2} \sum_{N \leq j \leq 2 N, j \neq j_{0}} \frac{c}{\left|j-j_{0}\right|}\left|\overline{\hat{F}_{j_{0}}(k)}\right|+C
\end{aligned}
$$

This term is dominated by (18). This completes the proof of (12).

\section{Remarks}

- The counterexample can easily be extended to larger $n$ (e.g. by appending some bump functions to the left or right of $F$ ).

- The counterexample above involved a potential $F$ which was bounded in $L^{2}$, but for which $\sup _{x}\left|T_{2}(F, F)(k, x)\right|$ and $\left|T_{3}(F, F, F)(k,+\infty)\right|$ were large (about $\log N)$ on a large subset of $[A, 2 A]$. By letting $N$ vary and taking suitable linear combinations of such variants of the above counterexample, one can in fact generate a potential $F$ bounded in $L^{2}$ for which $\sup _{x}\left|T_{2}(F, F)(k, x)\right|$ is infinite and $\left|T_{3}(F, F, F)(k, x)\right|$ accumulates at $\infty$ for $x \rightarrow \infty$ for all $k$ in a set of positive measure (one can even achieve blow-up almost everywhere). Thus it is not possible to estimate these multilinear expansions in any reasonable norm if one only assumes the potential to be in $L^{2}$. Similarly if $F$ had a derivative in $L^{2}$; it is the decay of $F$ which is relevant here, not the regularity.

- The unboundedness of $T_{3}$ on $L^{2}$ can be interpreted as stating that the (non-linear) scattering map $F \mapsto b_{k}(+\infty)$ from potentials to reflection coefficients is not $C^{3}$ on the domain of $L^{2}$ potentials. Similarly the map $F \mapsto a_{k}(+\infty)$ from potentials to transmission coefficients is not $C^{4}$ on 
the domain of $L^{2}$ potentials. In particular these scattering maps are not analytic.

- Despite the bad behavior of the individual terms $T_{k}(F, \ldots, F)$, the transmission and reflection coefficients $a_{k}(x), b_{k}(x)$ are still bounded for the counterexample given above. This phenomenon is similar to the observation that the function $e^{i x}=1+i x-x^{2} / 2-\ldots$ is bounded for arbitrarily large real $x$, even if the individual terms $(i x)^{n} / n$ ! are not.

We now sketch the proof of boundedness of $a_{k}, b_{k}$. Suppose that $k=$ $A j_{0} / N+O(1 / N)$ for some $N \leq j_{0} \leq 2 N$; we now fix $j_{0}$ and $k$. We can write

$$
\left(\begin{array}{c}
a_{k}(x) \\
b_{k}(x)
\end{array}\right)=G(x)\left(\begin{array}{l}
1 \\
0
\end{array}\right)
$$

where $G$ is the $2 \times 2$ matrix solving the ODE

$$
G^{\prime}(x)=\left(\begin{array}{ll}
0 & F(x) e^{-2 i k x} \\
F(x) e^{2 i k x} & 0
\end{array}\right) G(x) ; \quad G(-\infty)=\left(\begin{array}{ll}
1 & 0 \\
0 & 1
\end{array}\right) .
$$

We define the matrices $G_{j}$ similarly by

$G_{j}^{\prime}(x)=\left(\begin{array}{ll}0 & F_{j}(x) e^{-2 i k x} \\ F_{j}(x) e^{2 i k x} & 0\end{array}\right) G_{j}(x) ; \quad G_{j}(-\infty)=\left(\begin{array}{ll}1 & 0 \\ 0 & 1\end{array}\right)$.

We observe the identity

$$
G(x)=G_{j_{1}}(+\infty) G_{j_{1}-1}(+\infty) \ldots G_{N}(+\infty)
$$

whenever $N \leq j_{1} \leq 2 N$ and $x=N\left(j_{1}+\frac{1}{2}\right)$; this can be proven by an easy induction on $j_{1}$ and the observation that the above ODE are invariant under right-multiplication.

One can compute the $G_{j}(+\infty)$ using multilinear expansions (or using Gronwall's inequality), eventually obtaining

$$
G_{j}(+\infty)=\left(\begin{array}{ll}
1+\frac{i C}{j-j_{0}} & 0 \\
0 & 1-\frac{i C}{j-j_{0}}
\end{array}\right)+O\left(\left|j-j_{0}\right|^{-2}\right)
$$

for all $j \neq j_{0}$, where $C$ is a non-zero real constant. Because of the crucial factor of $i$ in the diagonal entries we see that the operator norm $\left\|G_{j}(+\infty)\right\|$ of $G_{j}$ is

$$
\left\|G_{j}(+\infty)\right\|=1+O\left(\left|j-j_{0}\right|^{-2}\right) .
$$

This allows one to multiply the $G_{j}(+\infty)$ together and obtain boundedness of $G(x)$ and hence $a_{k}(x), b_{k}(x)$.

In analogy with the observation concerning $e^{i x}$, one may need to use the fact that $F$ is real in order to obtain boundedness of eigenfunctions in the $L^{2}$ case. When $F$ is real there are additional estimates available, such as the scattering identity

$$
\int \log \left|a_{k}(+\infty)\right| d k=C \int|F(x)|^{2} d x
$$

for some absolute constant $C$; see for instance [5]. 
We do not yet know how to obtain boundedness of eigenfunctions for $L^{2}$ potentials $F$. However we have been able to achieve this for a model problem in which the Fourier phases $e^{2 i k x}$ are replaced by a dyadic Walsh variant $e(k, x)$. See [11].

- One can modify the counterexample to provide similar counterexamples for Schrödinger operators $-\frac{d^{2}}{d x^{2}}+V$ with $V \in L^{2}$, either by using the Miura transform $V=F^{\prime}+F^{2}$ mentioned in the introduction, or by inserting the standard WKB phase modification to the operators $T_{k}$ as in [2]. We omit the details.

- The multilinear expansion of $a$ leads to an expansion of $|a|^{2}$, whose quadratic term is equal to

$$
2 \operatorname{Re}\left(T_{2}(F, F)\right)=2 \operatorname{Re}\left(H_{-}\left(|\widehat{F}|^{2}\right)\right)=|\widehat{F}|^{2}
$$

This term is in $L^{1}$, which is better than the term $T_{2}(F, F)$, which is in general only in the Lorentz space $L^{1, \infty}$. The higher order terms of the expansion of $|a|^{2}$ are however unbounded again. Using the identity $|a|^{2}=$ $1+|b|^{2}$ we see that the fourth order term of $|a|^{2}$ is equal to

$$
2 \operatorname{Re}\left(\overline{T_{1}(F)} T_{3}(F, F, F)\right)
$$

We now define the modified potential

$$
G(x)=F(x)+G_{0}(x)
$$

where $F$ is as in the proof of Theorem 1.1 and $G_{0}(x)=\phi\left(x-N^{3}\right)$. Expanding the fourth order term by multilinearity, one observes that all terms can be estimated from above nicely with the exception of

$$
2 \operatorname{Re}\left(\overline{T_{1}(G)} T_{3}(F, F, F)\right)
$$

Since $T_{1}(G)=\widehat{G}$ has more rapidly changing phase than $T_{3}(F, F, F)$, the real part and the modulus $\overline{T_{1}(G)} T_{3}(F, F, F)$ are of comparable size on a large set, and so this term is of the order $\log (N)$ on a large set just like $T_{3}(F, F, F)$ itself.

\section{Acknowledgements}

The first author was supported by NSF grant DMS 0100796. The second author is a Clay Prize Fellow and is supported by a grant from the Packard Foundation. The third author was supported by a Sloan Fellowship and by NSF grants DMS 9985572 and DMS 9970469. The authors are grateful to M. Christ for pointing out the importance of the degeneracy in the phase function $\sum_{j}(-1)^{j} x_{j}$ and for suggesting numerous valuable improvements to the manuscript. 


\section{References}

[1] L. Carleson, On convergence and growth of partial sums of Fourier series, Acta Math. 116 (1966), 135-157.

[2] M. Christ, A. Kiselev, Maximal functions associated to filtrations, J. Funct. Anal. 179 (2001), 409-425

[3] WKB asymptotic behaviour of almost all generalized eigenfunctions of onedimensional Schrödinger operators with slowly decaying potentials, J. Funct. Anal. 179 (2001), 426-447.

[4] Scattering and wave operators for one-dimensional Schrödinger operators with slowly decaying nonsmooth potentials, to appear in Geom. Funct. Anal.

[5] P. Deift, R. Killip, On the absolutely continuous spectrum of one-dimensional Schrödinger operators with square summable potentials, Comm. Math. Phys. 203 (1999), 341-347.

[6] R. Hunt, On the convergence of Fourier series, 1968 Orthogonal Expansions and their Continuous Analogues (Proc. Conf., Edwardsville, Ill., 1967) pp. 235-255. Southern Illinois Univ. Press, Carbondale, Ill.

[7] M. Lacey, C. Thiele, $L^{p}$ estimates on the bilinear Hilbert transform for $2<p<\infty$, Ann. of Math. (2) 146 (1997), 693-724.

[8] _ On Calderón's Conjecture, Ann. of Math. (2) 149 (1999), 475-496

[9] C. Muscalu, T. Tao, C. Thiele, $L^{p}$ estimates for the biest I. The Walsh case, preprint.

[10] _ $L^{p}$ estimates for the biest II. The Fourier case, preprint.

[11] A Carleson type theorem for a Cantor group model of the scattering transform, Nonlinearity 16 (2003), 219-246.

[12] D. Pearson, Singular continuous measures in scattering theory, Comm. Math. Phys. 60 (1978), 13-36.

[13] C. Thiele, On the Bilinear Hilbert transform, Universität Kiel, Habilitationsschrift, 1998.

Department of Mathematics, UClA, Los Angeles, CA 90095-1555, U.S.A.

E-mail address: camil@math.ucla.edu

E-mail address: tao@math.ucla.edu

E-mail address: thiele@math.ucla.edu 